\title{
Surgical repair of the iatrogenic falsepassage in the treatment of trauma-induced posterior urethral injuries
}

\author{
Faruk Doğan $^{1}$, Ali Feyzullah Şahin ${ }^{1}$, Tevfik Sarıkaya ${ }^{2}$, Alper Dırık ${ }^{1}$ \\ ${ }^{1}$ Department of Urology, Şifa University Medicine School, Izmir, Turkey \\ ${ }^{2}$ Department of Urology, Sivas Public Hospital, Sivas, Turkey
}

\begin{abstract}
Summary
Pelvic fracture associated urethral injury (PFAUI) is a rare and challenging sequel of blunt pelvic trauma. Treatment of iatrogenic false urethral passage (FUP) remains as a challenge for urologists. In this case report we reviewed the iatrogenic FUP caused by wrong procedures performed in the treatment of a patient with PFAUI and the treatment of posterior urethral stricture with transperineal bulbo-prostatic anatomic urethroplasty in the management of FUP. A 37-year-old male patient with PFAUI had undergone a laparotomy procedure for pelvic bone fracture, complete urethral rupture, and bladder perforation 8 years ago. After stricture formation, patient had undergone procedures that caused FUP. Following operations, he had a low urinary flow rate, and incontinence and urgency even with small amounts of urine. FUP was diagnosed by voiding cystourethrography and retrograde urethrography. FUP was fixed with open urethroplasty with the guidance of flexible antegrade urethtoscopy. False passage should always be taken into account in the differential diagnosis of patients with persistent symptoms that underwent PFAUI therapy. In addition, we believe that in the evaluation of patients with PFAUI suspected for having a false passage, bladder neck and urethra should be assessed by combining routine voiding cystourethrography and retrograde urethrography with preoperative flexible cystoscopy via suprapubic route.
\end{abstract}

KEY WORDS: False urethral passage; flexible cystoscoby; pelvic fracture; urethral injury.

Submitted 30 July 2013; Accepted 31 December 2013

\section{INTRODUCTION}

Pelvic fracture associated urethral injury (PFAUI) is a rare and challenging sequel of blunt pelvic trauma. In 4$25 \%$ of male patients with pelvic bone fractures, simultaneous posterior urethral injury is observed (1). Injury to posterior urethra may be a simple contusion, or it may appear as partial or complete rupture (2). Urethral trauma may cause a life-long morbidity unless properly treated. While treatment approach in partial urethral rupture includes watchful waiting after performing a simple cystostomy, complete urethral rupture can be repaired with 3 methods: 1) Realignment of the separated urethral ends over a catheter (urethral realignment),

2) Primary anastomosis of separated urethral ends, and

3) Immediate suprapubic cystostomy and delayed repair of the resulting stricture on an elective basis (3).

Emergency treatment of PFAUI has not been standardized in developing countries. On majority of cases wrong procedures cause iatrogenic injuries in addition to trauma itself (4). Sometimes, careless and/or repeated urethral dilatations also lead to false urethral passage (FUP) formation, which results in infections and incontinence. Treatment of iatrogenic FUP remains as a challenge for urologists (5). Furthermore, there is a paucity of data about the urethroplasty procedure performed for urethral stricture formed by FUP. In this case report we reviewed the iatrogenic FUP caused by wrong procedures performed in the treatment of a patient with PFAUI and the treatment of posterior urethral stricture with transperinealbulbo-prostatic anatomic urethroplasty in the management of FUP.

Case Report and Figures are posted in Suppementary materials on www.aiua.it.

\section{Discussion}

False passage is an abnormal passage between urinary bladder and urethra, which is observed in association with posterior urethral stricture and is caused by a iatrogenic injury resulting from careless and wrong treatment procedures after trauma. If unnoticed, this false passage between urethra and bladder causes very frequent complaints; furthermore, repeated endoscopic procedures (internal urethrotomy), catheterization, or urethral dilatation become necessary. Following dilatation, hesitancy, incontinence, and urgency persist. Furthermore, false passage scar tissue around traumatized tissue causes prolonged and chronic infections and hence leads to stricture formation (6).

No conflict of interest declared 
Methods used for diagnosis of posterior urethral strictures before reconstruction should clearly delineate stricture length and site, determine the anatomy of posterior urethra and bladder neck, and show false passages and fistulas if any. Conventional methods include simultaneous CCUG and dynamic retrograde urethrography. It is necessary to make a pre-treatment assessment via combined radiological and endoscopic methods especially in obliterated cases where prostatic and proximal urethra cannot be visualized.

False passage is diagnosed with urethrography. Combined voiding cystourethrography and retrograde urethrography can clearly show the site and path of the passage (7). Cystourethroscopy plays a limited role in diagnosis since normal anatomical structures cannot be localized. This was also the case in our patient because his false passage could not be identified despite multiple endoscopic procedures at other medical facilities. A false passage should be taken into consideration for diagnosis in case normal anatomical markers like veru montanum, bladder trigone, and external sphincter could not be seen during cystourethroscopy. A flexible cystoscope advanced through a suprapubic route is highly useful for the diagnosis of false passage. While normal bladder neck is a funnel-shaped, soft, elastic, and of smooth structure, a false passage is a pale mucosal formation associated with a vertical circular dense scar and a coarse granulation that is located close to bladder neck (6).

In conclusion, recurrent urethral strictures develop due to inappropriate and insufficient initial therapies in patients with PFAUI with complete urethral separation;

\section{Figure.}

Preoperative VCUG and RUG shows the location of a false passage and urethral stricture. The black arrow shows normal bladder neck and prostatic urethra, the blue arrow shows the urethral stricture and the beginning of the intraprostatic false passage, while the white arrow shows the false passage located close to bladder neck.

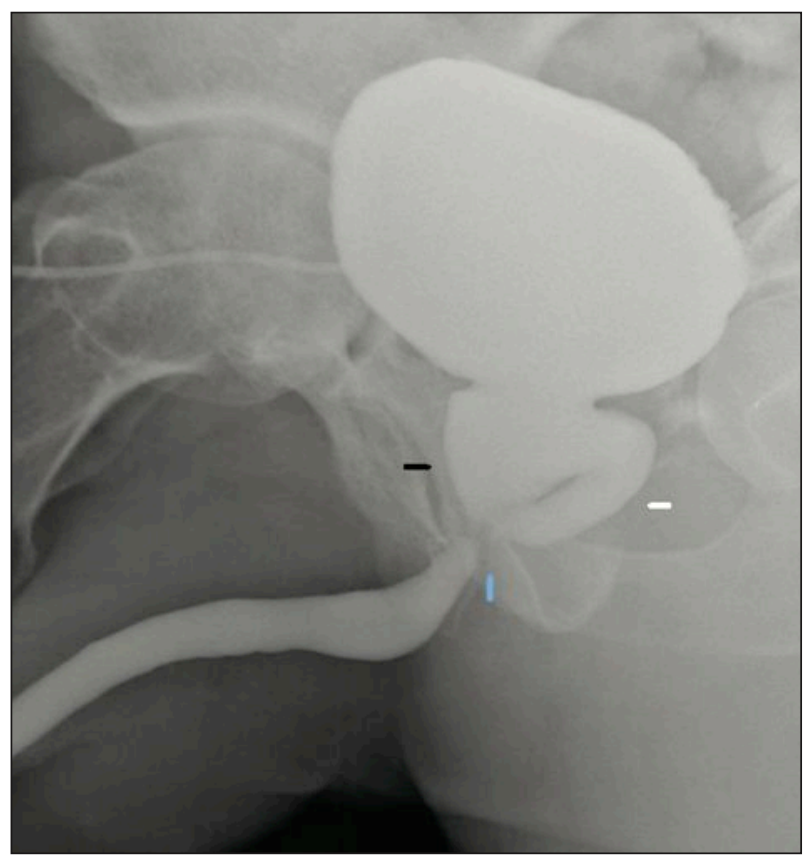

as a result, various endoscopic procedures and urethral dilatations become necessary. Even after these therapies, hesitancy, incontinence, and urgency are observed. False passage should always be taken into account in the differential diagnosis of patients with persistent symptoms that underwent PFAUI therapy. In addition, we believe that in the evaluation of patients with PFAUI suspected for having a false passage, bladder neck and urethra should be assessed by combining routine voiding cystourethrography and retrograde urethrography with preoperative flexible cystoscopy via suprapubic route.

The guidance of a flexible cystoscope via suprapubic route during the operation is quite helpful. It also guides procedures of curettage and dissection to determine the true anatomical structures and to identify the normal urethral tract, particularly in cases where prostatic urethra is blocked.

\section{REFERENCES}

1. Koraitim MM, Marzouk ME, Atta MA, et al. Risk of urethral injury in pelvic fractures. Br J Urol. 1996; 77: 876-80.

2. Lupu AN, Forrer JH, Smith RB, Kaufman J. Urethral gap in complete disruption of membraneus urethra. Urology. 1987; 29:378-82.

3. Webstre GD, Mathes GL, Selli C. Prostatomembranous urethral injuries: A review of the literature and a rational approach to their management. I. Urol. 1983; 130:898.

4. Barbagli G. History and evolution of transpubic urethroplasty: a lesson for young urologists in training. Eur Urol. 2007; 52:1290-2.

5. Barbagli G, Palminteri E, Lazzeri M, Guazzoni G. One-stage circumferential buccal mucosa graft urethroplasty for bulbous stricture repair. Urology. 2003; 61:452-5.

6. Qiang Fu, Jiong Zhang, Ying-long Sa, San-bao Jin, Yue-minXu. Transperineal bulbo-prostatic anastomosis for posterior urethral stricture associated with false passage: a single-centre experience. BJU Int. 2011; 108:1352-4.

7. Secrest CL. Staged urethroplasty: indications and techniques. Urol Clin North Am. 2002; 29:467-75.

\author{
Correspondence \\ Faruk Doğan, MD (Corresponding Author) \\ farukdogan58@gmail.com \\ Alper Dink, MD \\ a_dirik@yahoo.com \\ Specialist in Urology \\ Department of Urology, Şifa University Medicine School, Izmir, Turkey
}

Ali Feyzullah Sahin, MD, FEBU

ali.sahin@sifa.edu.tr

Asistant Professor in Urology

Department of Urology, Sifa University Medicine School

Sanayi cad. No:7 Bornova, Izmir, Turkey

Tevfik Sarkaya, MD

drts98@mynet.com

Specialist in Urology

Department of Urology, Sivas Public Hospital, Sivas, Turkey 